\title{
Probing the structure of local magnetic field of solar features with helioseismology
}

\author{
Khalil Daiffallah \\ Observatory of Algiers, CRAAG, \\ Route de l'Observatoire, BP 63, Bouzaréah 16340, Algiers, Algeria \\ email: k.daiffallah@craag.dz
}

\begin{abstract}
Motivated by the problem of local solar subsurface magnetic structure, we have used numerical simulations to investigate the propagation of waves through monolithic magnetic flux tubes of different sizes. A cluster model can be a good approximation to simulate sunspots as well as solar plage regions which are composed of an ensemble of compactly packed thin flux tubes. Simulations of this type are powerful tools to probe the structure and the dynamics of various solar features which are directly related to solar magnetic field activity.
\end{abstract}

Keywords. Magnetohydrodynamics, waves, magnetic field, sunspots, plages.

\section{Introduction}

Understanding the origin of the Sun's magnetic field is the most important topic in solar physics today. Sunspots are a manifestation of strong magnetic fields at the surface. Other magnetic features in the form of magnetic flux tubes can be distinguished, like plages which are concentrations of small-scale magnetic flux tubes (bright area in solar surface) and pores which are isolated vertical magnetic flux tubes. Constraining the subsurface structure, dynamics and evolution of these magnetic features is essential to establish a relationship between internal solar properties and magnetic activity in the photosphere. Helioseismology is a powerful tool to probe the structure and dynamics of the Sun through the observation of solar oscillations. However, the method of local helioseismology is still limited since the magnetic field is not included in the theory. We only interpret the observations in the quiet Sun in terms of temperature variation or velocity flow, but not in sunspots where there is a strong magnetic field. Therefore, numerical simulations are needed to infer the structure of the magnetic field by modeling the interaction of waves with magnetic features.

In the first part of this study (Section 2), we investigate the propagation of a linear surface gravity wave packet ( $f$-mode) through monolithic structures of magnetic flux tube of different sizes. In the second part (Section 3), we explore the helioseismic response of a cluster model which consideres the subsurface magnetic field of sunspots and plages as a bundle of small-scale magnetic flux tubes in a spaghetti-like configuration.

\section{Helioseismic signature of monolithic magnetic flux tubes}

We use the SLiM code (Cameron et al. 2007) to propagate a linear and Gaussian $f$-mode wave packet $(\nu=3 \mathrm{mHz})$ through a three dimensional enhanced polytropic atmosphere (Cally \& Bogdan 1997).

In this section, we explore the helioseismic response of magnetic flux tubes with radii from $200 \mathrm{~km}$ (e.g., internetwork magnetic field) to $3 \mathrm{Mm}$ (e.g., pores or small sunspots). We considere a $4820 \mathrm{G}$, purely vertical flux tube along the $z$-direction. The flux tube is 


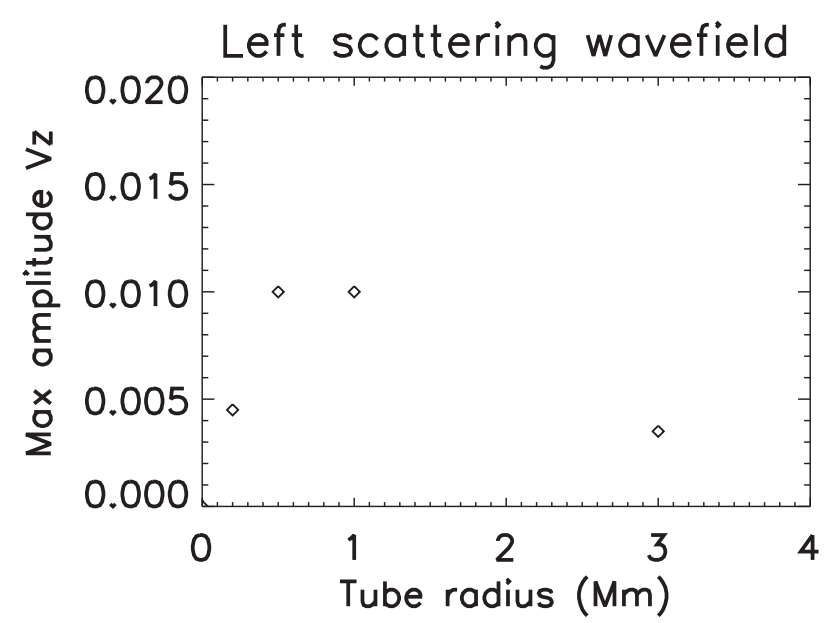

Figure 1. A plot of the maximum scattering amplitude as a function of tube radius measured in the far field.

almost evacuated and it is superposed on the background atmosphere. The scattered wave field is constructed as the difference between the simulation with and without the flux tube. Different scattered wave field patterns were observed for the different flux tubes. When the flux tube is small compared to the value of $\lambda / 2 \pi$ where $\lambda$ is the wavelength of the incident wave, mainly the $m=1$ kink modes are excited ( $m$ is the azimutal number of the wave). For mid-ranged tubes, the oscillations are a mixture of $m=1$ kink modes, and $m=0$ sausage modes. For larger tubes, numerous modes with various $m$ are excited (Daiffallah et al. 2011).

If we plot the maximum scattering amplitude versus tube radius measured in the far field (Fig. 1), we find that tubes with radii around $\lambda / 2 \pi$ are the largest scatterers. We explain the decrease of scattering after this value of radius by the excitation of $m$-modes and the absorption of waves by these large tubes. For example, in the case of tubes with $R=3 \mathrm{Mm}$, part of $f$-mode is converted to a slow magneto-acoustic-gravity mode which propagates along magnetic field lines in the $z$-direction (e.g., Cally 2005). This process can explain the observations of Braun et al.(1987) and Braun et al.(1988) which reveal a deficiency in the power of the outgoing wave compared to the incoming wave from a typical sunspot on the solar surface (Fourier-Hankel analysis).

\section{Helioseismic response of a cluster of small magnetic flux tubes}

In this section, we want to know what is the structure of the magnetic field beneath sunspots: is it like a monolithic model as in section 2 or like a cluster model? how to distinguish from the observed oscillations between these two models? The cluster model can be a good approximation to simulate solar plage regions which are composed of an ensemble of compactly packed thin flux tubes (Hanasoge \& Cally 2009).

Motivated by these problems, we investigate the propagation of waves ( $f$-mode) through a cluster of small identical magnetic flux tubes of $200 \mathrm{~km}$ radius (e.g., Hindman \& Jain 2012; Daiffallah 2013; Felipe et al. 2013).

We have studied two cases, one is a compact cluster which consists of seven identical magnetic flux tubes in a hexagonal close-packed configuration, and the other case is a loose cluster of nine tubes.

The Fig. 2 shows the horizontal displacement of the central tube axis as a function of depth $z$ (dot-dashed line) for the compact cluster in the left panel, and the loose 

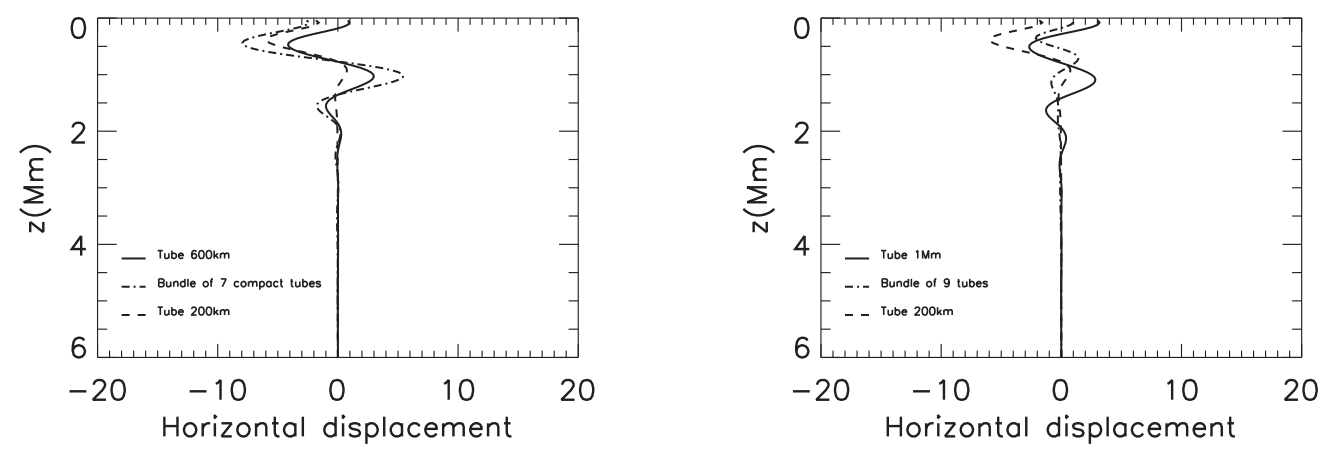

Figure 2. Horizontal displacement of the central tube axis of the compact cluster in the left panel, and the loose cluster in the right panel (dot-dashed line), as a function of depth $z$. The dashed and the solid lines show the oscillations of a single tube of $200 \mathrm{~km}$ radius and the monolithic equivalent tube, respectively. The separation between the tubes inside the loose cluster is about $\lambda / 2 \pi$.

cluster in the right panel. We can observe that the upper part of the compact cluster oscillates more like a single tube of $200 \mathrm{~km}$ radius (dashed line) than like the monolithic equivalent tube of $600 \mathrm{~km}$ (solid line). Furthermore, the large amplitude of the compact cluster compared to the amplitude of the $200 \mathrm{~km}$ single tube confirms that the oscillation concerns the totality of the compact cluster and not individual tubes. This can be seen in the scattered wave field of the compact cluster in the Fig. 3 where the compact cluster seems to oscillate like a single object and where there is no evidence of multiple scattering in the near field.

The amplitude of the loose cluster in the right panel of Fig. 2 is smaller than the amplitude of the $200 \mathrm{~km}$ single tube, which means that the incident wave energy is converted to tube oscillation and the oscillation of the loose cluster in Fig. 2 corresponds to that of the individual tubes. Therefore, the loose cluster will display multiple scattering from the individual tubes in the near field. In this case, the absorption of the incident wave by the cluster will be enhanced.

\section{Discussion}

Sunspots are manifestations of strong magnetic field at the solar surface. They represent a major relation between internal magnetic field and solar activity in the photosphere. Local helioseismology is a powerful tool to investigate the substructure of the Sun. However, interpretation of data have been somewhat ambiguous in solar active regions where the magnetic field is strong. Numerical simulations provide an efficient and direct way to understand the helioseismic signature of solar magnetic features, which have recently begun to be observed. We simulate in this study the propagation of a linear $f$-mode wave packet through different magnetic features. The aim is to get informations about the characteristics and the structure of these features by studying the scattered waves observed at the solar surface. The principal results of the simulations can be summarized in the following way:

- Magnetic flux tubes are strong absorbers and scatterers of $f$-mode waves.

- Different scattered wave field patterns were observed for different monolithic magnetic flux tube radii. This will allow us to infer the typical size of magnetic structures, but also magnetic field strength, vector orientation, profile, ...

- A cluster model of magnetic flux tubes (compact or loose) scatters waves in a different way from a monolithic model. This will allow us to infer the magnetic structure 


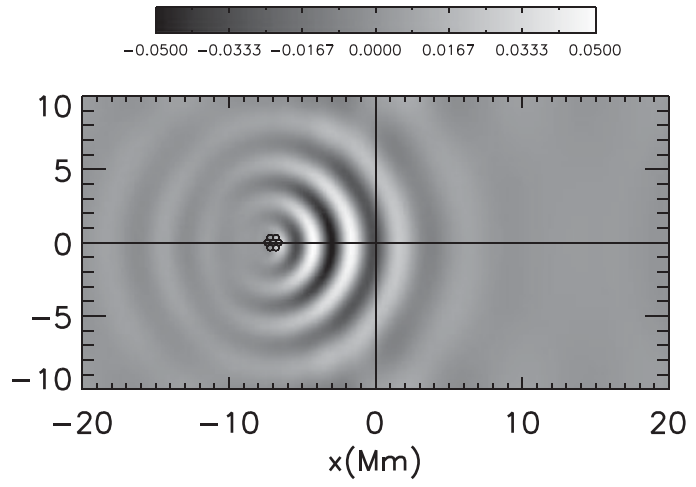

Figure 3. A snapshot at $t=3300 \mathrm{~s}$ of the scattered wave field (Vz) of a compact cluster of seven identical tubes of $200 \mathrm{~km}$ radius.

of more complex solar features (sunspots, plages, ...) and distinguish between monolithic or cluster models, compact or loose clusters.

- Determining the parameters of these magnetic features, that is, structure, typical size, field strength, ... will help to reveal details of the processes at work in the solar dynamo and how the magnetic field is transported up through the convection zone.

- The subsurface structure of sunspots is still poorly understood, highlighting the need for (i) high-resolution space observations, (ii) improved numerical simulations.

\section{References}

Braun, D. C., Duvall, T. L., Jr., \& LaBonte, B. J. 1987, ApJL, 319, L27

Braun, D. C., Duvall, T. L., Jr., \& LaBonte, B. J. 1988, ApJ, 335, 1015

Cally, P. S. \& Bogdan, T. J. 1997, ApJ, 486, L67

Cally, P. S. 2005, MNRAS, 358, 353

Cameron, R., Gizon, L., \& Daiffallah, K. 2007, AN, 328, 313

Daiffallah, K., Abdelatif, T., Bendib, A., Cameron, R., \& Gizon, L. 2011, Solar Phys., 268, 309

Daiffallah, K. 2013, Solar Phys., 212

Felipe, T., Crouch, A., \& Birch, A. 2013, ApJ, 775, 74

Giles, P. M., Duvall, T. L., Scherrer, P. H., \& Bogart, R. S. 1997, Nature, 390, 52

Hanasoge, S. M. \& Cally, P. S. 2009, ApJ, 697, 651

Hindman, B. W. \& Jain, R. 2012, ApJ, 746, 66 